\title{
Arthrosonography and Arthroscopy of Septic Carpitis in Donkeys (Equus Asinus)
}

\author{
Abdelhaleem. H. Elkasapy ${ }^{1{ }^{*}}$, Ibrahim. M. Ibrahim ${ }^{2}$, Samy.F. Ismail ${ }^{1}$, Badawy, A. \\ ${ }^{* \& 1}$ Department of Surgery, Faculty of Veterinary Medicine, Benha University \\ ${ }^{2}$ Department of Surgery, Anesthesiology and Radiology, Faculty of Veterinary Medicine \\ Cairo University \\ *abdelhaleem_elkasapy@yahoo.com \\ Abdelhalim.mahmoud@fvtm.bu.edu.eg
}

\begin{abstract}
Experimental septic arthritis was induced in the radiocarpal joint of 18 donkeys by intra-articular inoculation of Staphylococcus aureus $\left(3-4 \times 10^{6} \mathrm{CFU}\right)$. The inoculated animals were divided into three groups (6 donkeys in each group). The ultrasonographic and arthroscopic investigation was carried out before induction of septic carpitis and 3 days (group I), 14 days (group II), and 28 days ( group III) after induction of infection. The ultrasonographic findings of septic arthritis differ according to the period of induction. There is joint effusion associated with internal echoes with a variable echogenicity, ranging from anechoic to a mixed echogenicity especially in the third infected group.

The arthroscopic assessment of group I revealed hyperemia of synovial membrane and hypertrophied villi. In group II, severe hyperemia of synovial membrane, hypertrophied villi, pannus in the joint cavity and beginning of articular cartilage erosion were found. In group III, severe hyperemia of synovial membrane, hypertrophied villi, and more prominent articular cartilage erosion were present.
\end{abstract}

Keywords: Donkeys, Septic arthritis, Diagnosis, Ultrasonography, Arthroscopy.

\section{INTRODUCTION}

Septic arthritis can be caused by hematogenous corruption, traumatic injury, or itrogenic infection (Honnas, Schumacher, and Kuesis, 1992) In adult horses, direct trauma is the most common cause of infectious arthritis where tissue destruction and cellulitis can lead to an open joint and subsequent infectious arthritis (McIlwraith, 1987). The most common microorganism isolated from donkeys suffering from septic carpits was Staphylococcus aureus (El-Maghraby and Al-Bawa'neh 2002)

Ultrasonography is a useful imaging modality for the investigation of joint abnormalities as it enables the estimation of soft tissue components of the joint and provides information on the regularity of the bony contours (Redding, 2001; Smith, 2008).

The transducer of the highest frequency could maintain adequate depth penetration. The highfrequency transducers produce superior spatial and contrast resolution, while the low-frequency transducer improves tissue penetration (Nyland et al., 1995)

The ultrasonography is highly accommodating in the detection of carpal soft tissue abnormalities in horses. The extensor carpi-radialis and the common digital extensor tendons, were the most easily visualized structures. They added that the ultrasonography is ideal to evaluate the soft-tissue structures, surrounding the articualr regions, e.g. the equine carpus (Tnibar et al., 1993; Ross, 2003).

Ultrasonographic examination of the carpus revealed that the extensor tendons were normally imaged over the dorsal aspect of the carpal joints. The extensor carpi radialis tendon was echogenic with a parallel fiber pattern and was surrounded by a synovial sheath containing a very small amount of anechoic fluid. The common and lateral digital extensor tendons both have synovial sheaths. The extensor carpi obliquus tendon is also surrounded by sheath (Reef, 2004).

Ultrasonographic examination of the tarsocrural joint in septic arthritis. showing accumulation of anechoic fluid filled with hypoechoic dots within the joint capsule (AbdEl-Glil, 2012).

Arthroscopy provides a far more thorough examination of the carpal joints than arthrotomy by effectively moving the illumination and the examiners eye into the joint. Complete inspection of the joint with the use of an arthroscope cannot be emulated by any arthrotomy incisions (Mcllwraith et al, 2005). 
Magda, El-Rashidy, Saleh and Makady (2005) stated that the arthroscopic examination of the radiocarpal joint of the donkey from the lateral portal revealed that the medial joint angle formed by the distal surface of the radius and the proximal surface of the radial carpal bone was first seen. They also added that arthoscopic examination of the radiocarpal joint from the medial portal revealed that the first area seen was the lateral joint angle formed by the proximal articular surface of the intermediate carpal bone and the distal articular surface of the radius.

Arthroscopy is considered the most valid method for cartilage evaluation, but in case of small cartilage lesions there is a risk of overestimation of the defect size. The advantage of arthroscopy compared to the other diagnostic modalities is the possibility of immediate treatment of the identified joint problem (Niemeyer, Pestka, Erggelet, Steinwachs, Salzmann and Sudkamp, 2011; Spahn, Klinger, Baums, Pinkepank and Hofmann, 2011).

Adequtae arthroscopic examination of the intercarpal or radiocarpal joint is possible through a single dorsal arthroscopic portal for each joint. Using two dorsal separate portals improves visualization by increasing freedom of movement because of reduced soft tissue tension around the arthroscope and a reduced tendency to slip out of the joint when examining areas close to the arthroscopic portal (McIlwraith et al, 2005).

Acute synovial infection is characterized arthroscopically by severe synovitis. Established infection frequently results in the production of an intrasynovial fibrinocellular conglomerate (pannus). This may cover the foreign material and devitalised tissues, act as a nidus for bacterial multiplication and was rich in inflammatory cells, degradative enzymes, and radicals. It is also a barrier to synovial membrane diffusion, thus compromising further intrasynovial nutrition and limiting access for circulating antimicrobial drugs. The quantum and the nature of the pannus appears to be dependent on the type and number of infecting organisms (Wright, 2002).

Arthroscopic examination of septic arthritis of the tarsocrural joint in horse showing erosions and irregularity of articular surface, fissure, articular degeneration and hypertrophied villi (AdbEl-Glil, 2012).

Borg and Carmalt (2013) concluded that joint infection rate in the horse population that had elective arthroscopy without antimicrobial prophlaxsis compared favorably with other reports citing $0.9 \%$ sepsis in horses after arthroscopy.

This study aimed to describe the arthroscopic anatomy of the radiocarpal joint in donkeys, and to through a light and join the ultrasonographic findings and arthroscopic assessment of induced septic arthritis (Staphylococcus aureus) of the radiocarpal joint in donkeys.

\section{MATERIALS AND METHODS}

The present study was experimentally induced on 32 healthy, adult donkeys of both sexes. Fourteen animals were used for serial propagation of the Staphylococcus aureus untill the infective inoculums were obtained. The remaining 18 donkeys were divided into three groups each group consists of 6 animals. In these animals, induction of septic carpitis in the radiocarpal joints of donkeys by Staph. Aureus was induced. After the induction of septic carpitis arthroscopic examination were recorded. These animals were divided into three groups. The examination of the three infected groups was applied 3, 14 and 28 days after induction of septic carpitis of the radiocarpal joint.

\subsection{Induction of Septic Arthritis (Septic Carpitis)}

The induction of septic arthritis in donkeys was performed by using of a viable Staphylococcus aureus colony (standard colony). Staphylococcus aureus used for induction of septic arthritis in this study was isolated from a donkey suffering from septic arthritis. A sample from the infected synovial fluid of the carpal joint was aspirated, inoculated in nutrient broth and incubated at 24 hours at $37^{\circ} \mathrm{C}$. Each radiocarpal joint was inoculated with 3 to $4 \times 10^{6}$ colony-forming units of viable Staphylococcus aureus.

\subsection{Ultrasonographic Examination}

Ultrasonographic examination was performed with a mobile ultrasound machine(LOGIQ TM 180 Medical System)(7.6 MHZ linear probe) in the faculty of veterinary medicine Benha university and a real time Toshiba company ultrasound system(Toshiba Medical Company ultrasound system ), using multi frequency probes ( 7 micro convex and $8 \& 10 \mathrm{MHZ}$ linear probe) in the faculty of veterinary 
medicine, Cairo university with displayed depth of $4-6 \mathrm{~cm}$. The scans were frozen and photographs were taken on Polaroid films in both longitudinal and transverse scans. The hair over the carpal region was clipped and shaved, and then sonographic contact gel was applied on the skin.

\subsection{Diagnostic Arthroscopy of the Carpal Joints}

\subsubsection{Anesthesia of Donkeys for Arthroscopic Surgery}

Arthroscopic examination was performed on donkeys under the effect of XylazineHcl $(1 \mathrm{mg} / \mathrm{kg})$ as tranquilizer and thiopental sodium (5\% solution intravenously in a dose of 6-8 $\mathrm{mg} / \mathrm{kg}$ body weight).

\subsubsection{Preparation of the Carpus for Arthroscopy}

The carpus was clipped circumferentially from the proximal metacarpus to the distal radius, cleaned and draped with sterile towel immediately prior to surgery. The horse or donkey was placed in dorsal recumbency and hooves were suspended on bar for ease of control of the degree of flexion of the joint.

\subsubsection{Arthroscopic Procedures}

The arthroscope was inserted in the radiocarpal joint while the joint was 20 degree flexed. Two arthroscopic portals (lateral and medial) were used for each joint. The lateral portal was located half way between the tendons of the extensor carpi radialis and the common digital extensor tendon. The medial portal was located about $1 \mathrm{~cm}$ medial to the tendon of extensor carpi radialis. Distension of the joint was obtained with $15-20 \mathrm{ml}$ of 0.9 normal saline using an 18 gauge needle inserted at the proposed site for insertion of the arthroscope mentioned above. The arthrosopic sleeve was inserted through a $1 \mathrm{~cm}$ skin incision at the site of needle insertion. With the aid of a sharp obturator, the sleeve was advanced into the joint until the fluid began to escape from the canula. The sharp obturator was replaced with a $25 \& 4 \mathrm{~mm}$, forward oblique viewing arthroscope. An ingress fluid line and fiber optic light cable were attached to the arthroscope. The joints were viewed with a video camera and monitor. The joints were irrigated throughout the procedure with normal saline delivered from an infusion set.

\section{RESULTS}

The serial propagation of Staphylococcus Aureus in donkey's carpal joints revealed that the infective dose of septic carpitis was $3-4 \times 10^{6} \mathrm{CFU}$.

\subsection{Normal Ultrasonographic Findings}

The longitudinal ultrasonographic scanning of the common digital extensor tendon (CDET) and the extensor carpi radialis tendon (ECRT) was performed on the dorsolateral (CDET) and dorsomedial(ECRT) aspects of the carpal joint. Both tendons appeared as parallel linear hyperechoic structure. ECRT size was larger than CDET.

The transverse image of the common digital extensor tendon (CDET) and the extensor carpi radialis tendon (ECRT) appeared ultrasonographically as an elliptical to oval echogenic in shape structure and ECRT had a larger size than CDET.

The ultrasonographic examination of the radiocarpal joints revealed that the synovial fluid appeared as a small anechoic area surrounded by hyperechoiccapsule (Fig. 1).

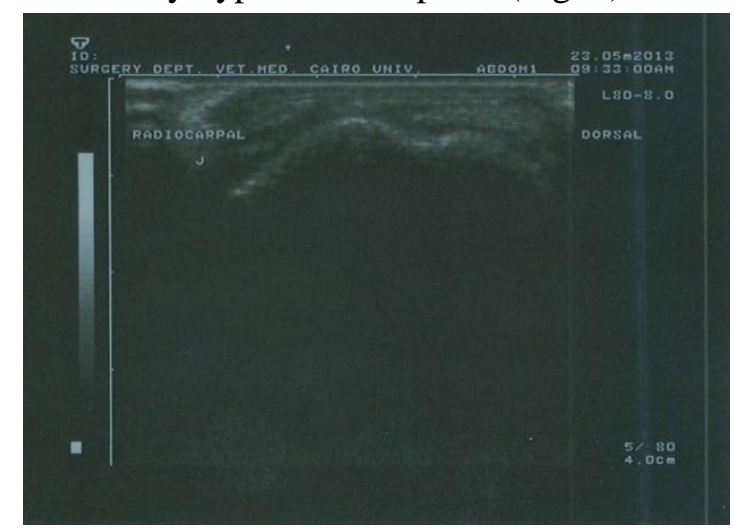

Fig(1). ultrasonographic image of radiocarpal joint in normal donkey showing a small anechoic area surrounded by hyperechoic capsule. 
A longitudinal ultrasonographic evaluation of the common digital extensor tendon (CDET) in the infected groups I, II and III revealed anhyperechoic image of mild to moderate disturbed of linear organization of the tendon fiber. The same findings was found in sonographic evaluation of the extensor carpi radialistendon (ECRT).

In cross section, the ultrasonographic image of the CDET and ECRT was composed of a highly echogenic, compact stippled echopattern, nearly to the normal tendon.

\subsection{Ultrasonographic Findings of the Three Infected Groups}

In the sonographic evaluation of the radiocarpal joint of the infected three groups of animals, it was found a variable huge amount of anechoic synovial fluid accumulation (joint effusion) with mild hyperechoic non shadowing echogenicity (fibrinous shadow). Also, thick hyperechoic joint capsule was showed (Fig. 2, $3 \& 4$ ).

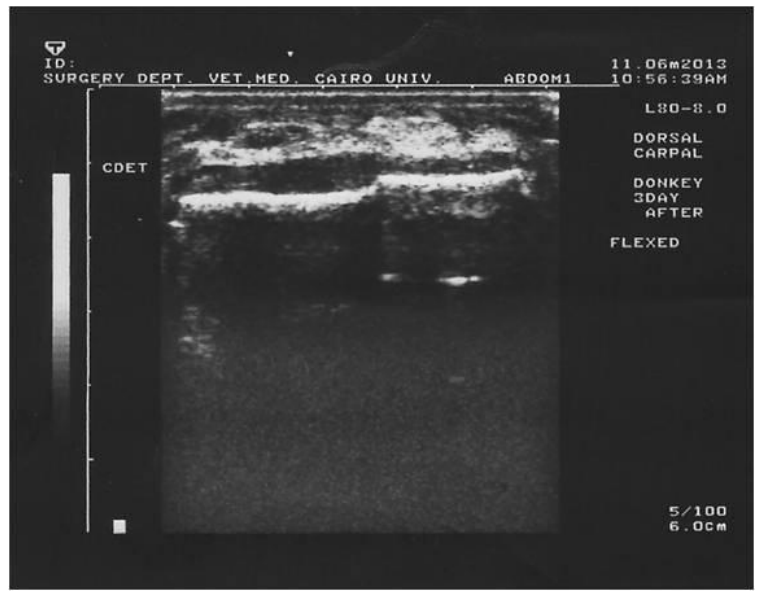

Fig(2). ultrasonographic image of radiocarpal joint in group I showing large anechoic area surrounded by hyperechoic capsule.

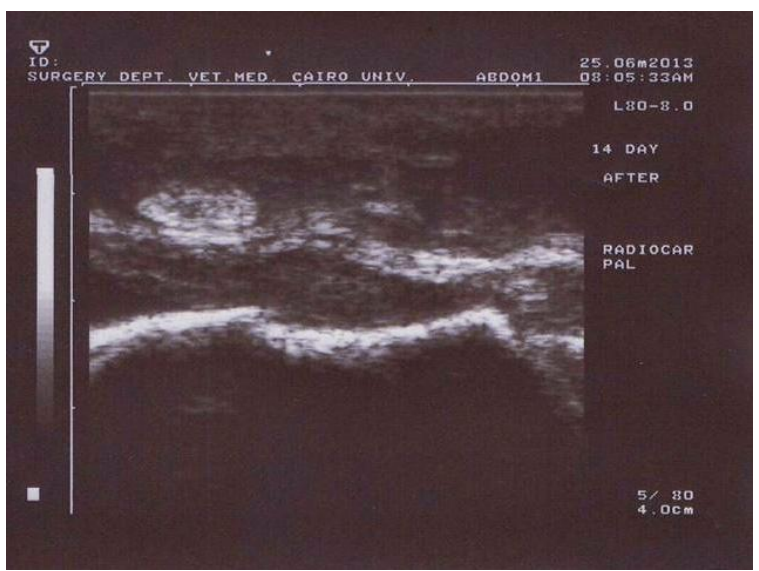

Fig(3). ultrasonographic image of radiocarpal joint in group II showing large anechoic area filled with hypoechoic dots which represent the fibrinous content of the synovial fluid surrounded by hyperechoic capsule.

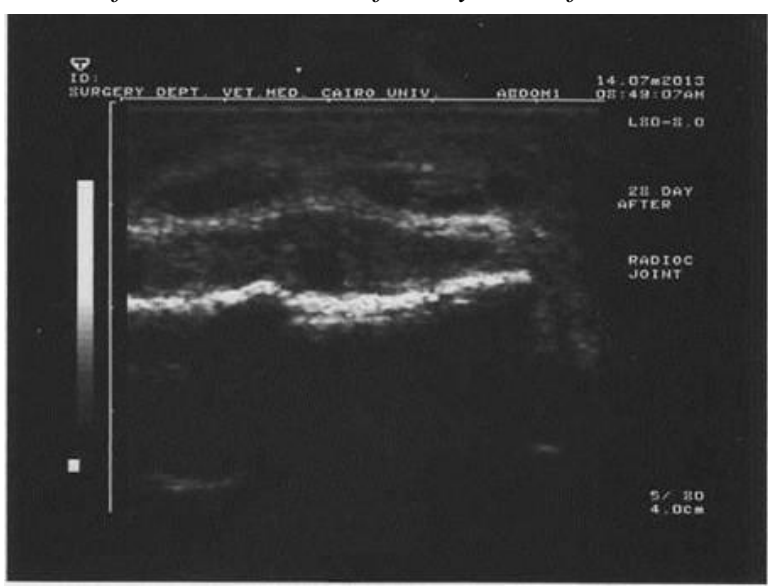

Fig(4). ultrasonographic image of radiocarpal joint in group III showing large anechoic area filled with hypoechoic dots which represent the fibrinous content of the synovial fluid surrounded by hyperechoic capsule. 


\subsection{Normal Arthroscopic Examination of Radiocarpal Joint}

\subsubsection{Arthroscopic Examination of the Radiocarpal Joint from the Lateral Portal}

Arthroscopic examination of the radiocarpal joint from the lateral portal revealed that the medial joint angle formed by the distal surface of the radius and the proximal surface of the radial carpal bone was the first area seen (Fig, 5). Withdrawing of the arthroscope makes easier the visualization of the proximal surface of the intermediate carpal and the distal surface of the radius in its middle portion could be easily seen. The lateral joint angle could be seen from this portal with some difficulties, but it was better examined from the medial portal.

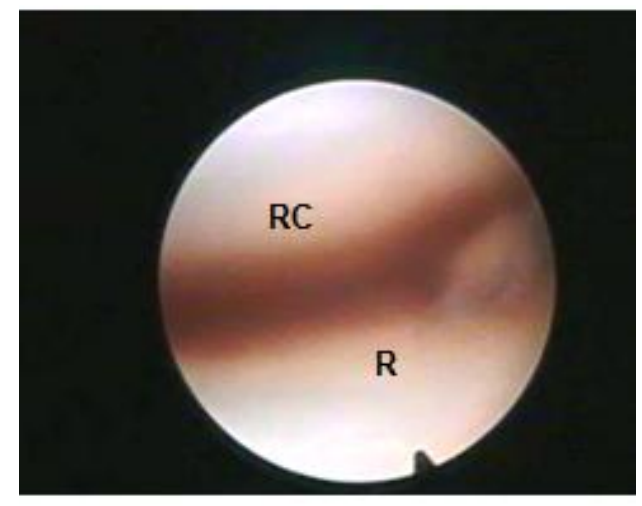

Medial portion of $R C J$

Fig(5). Arthroscopic view of the radiocarpal joint showing the medial joint angle. $(R)$ radius, $(R C)$ radial carpal bone.

\subsubsection{Arthroscopic Examination of the Radiocarpal Joint from the Medial Portal}

Arthroscopic examination of the radiocarpal joint from the medial portal revealed that the first area seen was the lateral angle formed by the proximal articular surface of the ulnar carpal bone, the lateral aspect of the intermediate carpal bone and the distal articular surface of the radius (Fig, 6). The joint space, synovial membrane and villi were easily examined. The shape of the villi varied from polyp like (Fig, 7), slender, stunted and finger like

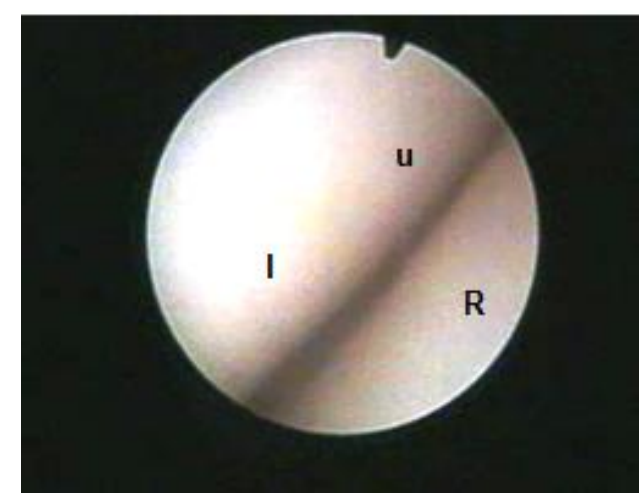

lateral portion

Fig(6). Arthroscopic view of the radiocarpal joint showing the lateral joint angle. $(R)$ radius, $(u)$ ulnar carpal bone, (I) intermediate carpal bone.

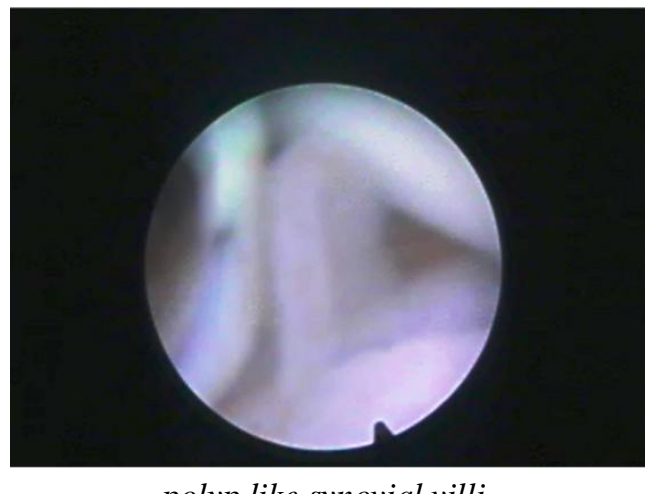

polyp like synovial villi

Fig(7). Arthroscopic view of the radiocarpal joint showing polyp like synovial villi 


\subsubsection{Arthroscopic Examination of Group One}

Arthroscopic examination of infected group one revealed that the cartilage remained clear and did not change. Mild degree of synovitis which characterized by hyperemia and petechiation of the villi (Fig, 8), slight degree of congestion and hypertrophied villi are present.

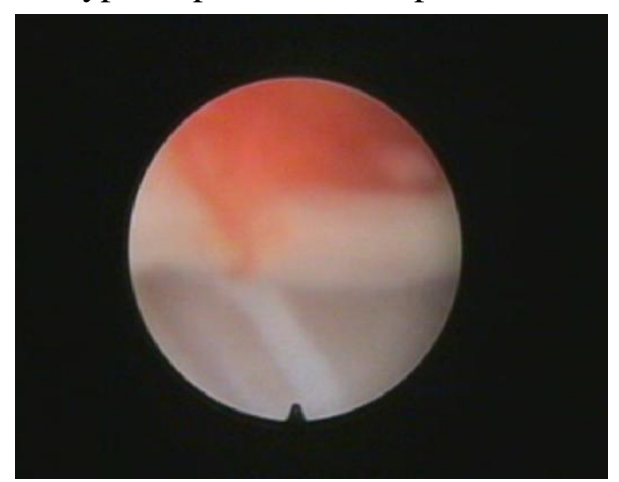

Congestion in $G 1$

Fig(8). Arthroscopic view of the radiocarpal joint of a donkey from group one showing congestion of the synovial villi.

\subsubsection{Arthroscopic Examination of Group Two}

Arthroscopic examination of infected group two revealed the presence of a sever degree of synovitis which was characterized by hyperemia and petechiation of the villi. Sever degree of congestion and hypertrophied villi were present. Pannus (intra synovial fibrinocellular conglomerate) was present at this stage in addition to, beginning of articular cartilage erosion (Fig, 9).

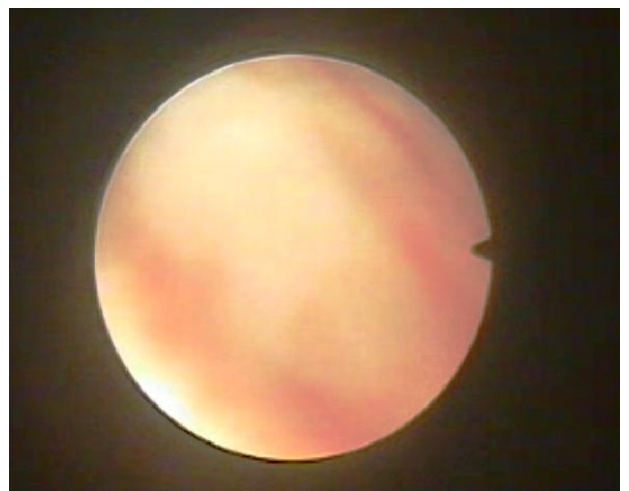

Pannus in $G 2$

Fig(9). Arthroscopic view of the radiocarpal joint of a donkey from group two showing pannus

\subsubsection{Arthroscopic Examination of Group Three}

Arthroscopic examination of infected group three revealed the presence of a more severs degree of synovitis characterized by hyperemia and petechiation of the villi. Sever degree of congestion which appear as patches, hypertrophied villi and more prominent erosion of articular cartilage was present (Fig, 10).

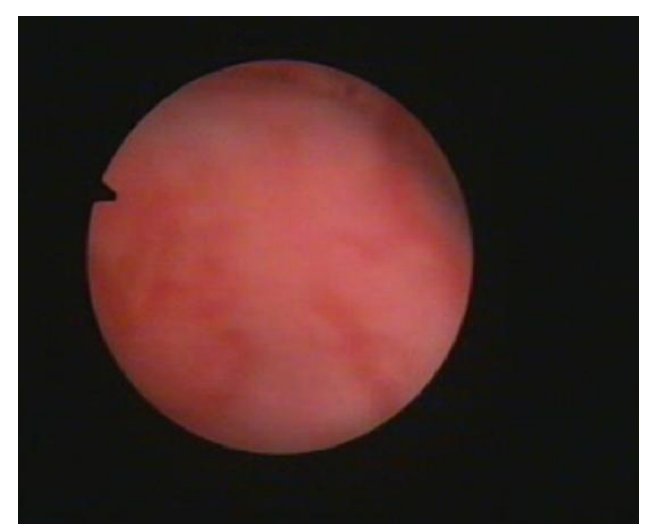

Erosion in $G 3$

Fig(10). Arthroscopic view of the radiocarpal joint of a donkey from group three showing more prominent erosion of the articular cartilage. 


\section{DISCUSSION}

In the present study, Staphylocucus Aureus was used in the induction of septic carpitis in donkeys as the most commonly isolated micro-organisms from clinical case was this micro-organism, this study was performed in donkeys to state the severity of every stage of septic carpitis and furthermore, use it on the clinical trails. These findings agree with that reported by El-Maghraby and Al-Bawa'neh (2002).

Ultrasonographic examination of common digital extensor tendon (CDET) and extensor carpi radialis tendon (ECRT) was easily performed on the dorsal aspect of the carpal joints. These findings agree with (Reef, 2004; Morsey, 2011; El-Kasapy, 2013).

Ultrasonographic examination of septic carpitis appeared as marked distension of the joint with anechoic synovial fluid which filled with hypoechoic dots which represent the fibrinous content of the synovial fluid. These findings agree with (Reef, 1998; AbdEl-Glil, 2012; El-Kasapy, 2013; El-Kasapy et al, 2013)

The obtained results revealed that, the arthroscopic examination of the all aspects of the radiocarpal joint was accomplished through medial and lateral portals. This finding agrees with Magda et al (2005) and El-Kasapy et al, (2014). However, Martin and Macllwraith (1985); MacIlwraith (1990) and MacIlwraith et al (2005) recorded that adequate arthroscopic examination of the radiocarpal joint was possible through a single arthroscopic portal. Using two separate portals, in the present study improved visualization of all joint structures by increasing the freedom of movement of arthroscope inside the joint cavity, reducting of soft tissue tension around the arthroscope and its tendency to slip out of the joint.

The lateral portal of the radiocarpal joint was positioned halfway between the extensor carpi radialis tendon and common digital extensor tendon at the level of the radiocarpal joint. The medial portal was located medial to the extensor carpi radialis tendon at the level of the radiocarpal joint. This finding agrees with Magda et al, (2005). The same portals also were described by Martin and MacIlwraith (1985); Hurtig and Fertz (1986); MacIlwraith et al, (1987); MacIlwraith (1990) and MacIlwraith et al, (2005) in horses. Arthroscopic insertion at this level was save to the neurovascular in the joint area.

Arthroscopic examination of the radiocarpal joint was best achieved with the joint flexed at 20 degree. The same finding was recorded by Magda, et al (2005) in donkeys and also by Martin and MacIlwraith (1985) and MacIlwraith et al, (2005) in horses. Arthroscopic examination of the joint flexed at 20 degree reduces the tension of the synovial membrane on the dorsal aspects of the articular surfaces of the radial carpal bone, intermediate carpal bone and the radius.

Arthroscopic examination in cases of septic arthritis revealed synovitis which was characterized by hyperemia and petechiation of the villi. This finding agrees with Macllwraith (1984) who illustrated that synovitis was characterized by hyperaemia, petechiation of the villi, development of small hyperaemic villi in abnormal locations and new forms of villi. With severe inflammation, fusion of villi and the presence of fibrinoid strands may be observed.

Arthroscopic examination of septic carpitis (group two) revealed the presence of a sever degree of synovitis with hyperemia and petechiation of the villi. Sever degree of congestion and hypertrophied villi were present. Pannus was present at this stage in addition to the presence of erosion of the articular cartilage. These findings agree with MacIlwraith (1984); Wright (2002); AdbEl-Glil (2012) El-Kasapy et al, (2014).

Arthroscopic examination of septic carpitis (group three) revealed presence of more sever degree of synovitis characterized by hyperemia and petechiation of the villi. Sever degree of congestion which appear as patches, hypertrophied villi and erosion were also present. These findings agree with MacIlwraith (1984) and AdbEl-Glil (2012).

In conclusion, ultrasonography and arthroscopy provides advantages of diagnosis and management of infected joint to choose the suitable method of treatment.

\section{REFERENCES}

AbdEl-Glil, A.I. 2012. Studies on equine tarsal region: diagnostic imaging and management. $\mathrm{PhD}$ thesis, Faculty of Veterinary Medicine Cairo University.

Borg, H. and Carmalt, J.L. 2013. postoperative septic arthritis after elective equine arthroscopy without antimicrobial prophlaxisis. Vet. Surgery (42):3:262-266. 
El-Kasapy, A.H. 2013.Studies on Equine Carpitis. PhD thesis, Faculty of Veterinary Medicine Benha University.

El-Kasapy, A.H., Badawy, A.M, Ismail, S.F. and Ibrahim, I.M. 2013. Radiographic and Ultrasonographic Diagnosis of Septic Carpitis in Equine: An experimental study. Benha Vet. Medical J. 25 (1):239-247.

El-Kasapy, A.H., AbdelGalil, A.I., Al-Akraa, A.M, Ibrahim, I.M. and Ismail, S.F. 2014. Arthroscopy of septic carpitis in donkeys (Equus asinus). Open Veterinary Journal, 4(2): 113-117.

El-Maghraby, H.M. and Al-Bawa'neh, H.A. 2002. Evaluation of sodium hyalournate for the treatment of experimentally induced infectious arthritis in donkeys. J. Egypt. Vet. Med. Ass. 62 no (6a) pp:53-69.

Honnas, C.M.; Schumacher, J. and Kuesis, B.S. 1992. Ankylosis of the distal interphalangeal joint in a horse after septic arthritis and septic navicular bursitis. J. Am. Vet. Med. Assoc. Vol. 200 No.7; pp' 964-968.

Hurtig, M.B. and Fertz, P.B. 1986. Arthroscopic landmarks of the equine carpus, J.A.V.M.A. 189(10):1314-1321.

Macllwraith, C.W. 1984. Experiences in diagnosis and surgical arthroscopy in the horse. Equine Vet. J. 16(1):11-19.

McIlwraith, C. W. 1987. Diseases of the joints, tendons, ligaments and related structures. In: Stashak, T.S., ed. Adams' Lameness in horses, 4th edition. Lea \& Febiger, Philadelphia; pp. 339-447.

MacIlwraith, C.W.; Yovich, J.V. and Martin, G.S. 1987. Arthroscopic surgery for the treatment of osteochondral chip fractures in the equine carpus. J.A.V.M.A. 191(5):531-540.

Magda, M. A., El-Rashidy, M.H., Saleh, A.S., and Makady, F.M. 2005. Arthroscopic anatomy of the carpal joint in donkey. 2nd international scientific conf., April 26-28. 2005. Qena\&Luxor, Egypt.

Martin, G.S. and McIlwraith, C.W. 1985. Arthroscopic anatomy of the intercarpal and radiocarpal joints of the horse. Equine Vet.J.17(5):373-376.

McIlwraith C.W., Nixon A.J., Wright I.M., Boening K.J. 2005. Diagnostic and surgical arthroscopy of the carpal joints. In: McIlwraith C.W., Nixon A.J., Wright I.M., Boening K.J. (editors). Diagnostic and Surgical Arthroscopy in the Horse. $3^{\text {rd }}$ edition, Elsevier, Philadelphia.

Mcllwraith, C.W. 1990. Diagnostic and surgical arthroscopy in the horse.. 2nd ed. Philadelphia. Lea \&Febiger.

Niemeyer P., Pestka J.M., Erggelet C., Steinwachs M., Salzmann G.M., Sudkamp N.P. 2011. Comparison of arthroscopic and open assessment of size and grade of cartilage defects of the knee. Arthroscopy 27, 46-51.

Nyland, T.G., Mattoon, J.S. and Wisner, E.R. 1995. Physical principles instrumentation and safety of diagnostic ultrasound. In: Nyland TG, Mattoon JS eds. Veterinary Diagnostic Ultrasound. Philadelphia. W.B. Saunders Co., 3-18.

Ross, M. W. 2003. The carpus. In: Diagnosis and Management of Lameness in the Horses, Eds: M. W. Ross and S. J. Dyson, W. B. Saunders Co. ,Philadelphia. Pp612-619.

Redding, W.R. 2001. Use of ultrasonography in the evaluation of joint disease in horses. Part 1: indications, technique and examination of the soft tissues. Equine Veterinary Education 13(4):198-204.

Reef, V.B. 2004. Joint ultrasonography. Clin Tech Equine Pract 3:256-267 (C) Elsevier Inc.

Smith, R. 2008. Using ultrasound to image joints. In: 10th International Congress of World Equine Veterinary Association. Moscow, Russia. Pp: 279-282.

Spahn G., Klinger H.M., Baums M., Pinkepank U., Hofmann G.O. 2011. Reliability in arthroscopic grading of cartilage lesions: Results of a prospective blinded study for evaluation of interobserver reliability. Archives of Orthopaedic and Trauma Surgery 131, 377-381.

Tnibar, M.A., Kaser-Hotz, B. and Auer, J.A. 1993. Ultrasonography of the dorsal and lateral aspect of the equine carpus: Technique and normal appearance. Vet. Radiol. Ultrasound, 34 (6): 413-425.

Wright, I.M. 2002. Arthroscopic surgery in the management of contamination and infection of joints, tendon sheaths, and bursae. Clinical Techuiques in Equine Practice, Vol 1, No, 4, 2002, pp:234244. 